\title{
Stiffness Characteristics Analysis of Interconnected Hydro-Pneumatic Suspension for Heavy Multi-Axle Vehicles
}

\author{
Xu Wang ${ }^{a}$, Qinhe Gao ${ }^{b}$, Zhihao Liu ${ }^{\mathrm{c}}$ and Jiangtao Feng ${ }^{\mathrm{d}}$ \\ Xi'an High-Tech Research Institute, Xi'an Shaanxi 710025, China. \\ a1219939540@qq.com, b gao202@189.com, c1066180281@qq.com, d fengjt291082217@126.com
}

Keywords: Interconnected hydro-pneumatic Suspension, Stiffness characteristics, Nonlinear, Riding comfort.

\begin{abstract}
With heavy multi-axle vehicle interconnected suspension system as research object, a mathematical model of elastic force of four-axis interconnected hydro-pneumatic suspension system is established and simulation and bench test are carried out. Based on the mathematical model, the stiffness characteristics of interconnected suspension and independent suspension under different working conditions are compared and analyzed, and the stiffness characteristics of interconnected suspension are also parametrically analyzed. The results show that compared with independent suspension, the stiffness of interconnected suspension system is increased and the nonlinear characteristics are obvious under single wheel excitation and rolling conditions. Each parameter has different influence on stiffness characteristics. The vertical stiffness increases with the increase of the diameter of piston rod, the increase of the initial pressure and the decrease of the initial volume of accumulator. The roll stiffness increases with the increase of the diameter of the cylinder, the decrease of the diameter of piston rod, the decrease of initial volume and the increase of initial pressure of accumulator, and the increase of installation distance of two cylinders
\end{abstract}

\section{Introduction}

As large loading and transportation vehicles, heavy multi-axle vehicles are necessary for the development of military vehicles and off-road vehicles [1]. Heavy multi-axle vehicle mostly adopts hydro-pneumatic suspension to improve riding comfort and stability. The interconnected hydro-pneumatic suspension system is widely used in heavy multi-axle vehicles now because of its nonlinear stiffness, balancing the load of each axle, anti-roll and so on, which is superior to independent suspension [2].

Domestic and foreign scholars have conducted extensive research on hydro-pneumatic suspension system. CAO et al. studied the roll stiffness, pitch stiffness and damping characteristics of interconnected suspension system with different modes [3]. Smith et al. studied the dynamic characteristics of an off-road vehicle equipped with hydraulic interconnected system [4]. Wang Zeng Quan, et al. considering the factors of oil compression and piston rod friction, established a nonlinear model of interconnected hydro-pneumatic suspension, and verified by test [5]. Zhang Junwei established the mathematical model of interconnected suspension system under multiple working conditions and analyzed the system characteristics [2]. Chen Sheng Zhao et al. designed a rolling interconnected hydraulic suspension system, established a mechanical-hydraulic coupling vehicle dynamics model, and carried out tests to analyze the effect of key parameters on vehicle [6]. Ding Fei et al. deduced the impedance matrix of hydraulic subsystem based on transfer matrix method, established the multi-body dynamic equation and studied the vibration characteristics of the car body [7].

Based on the research of characteristics of two-axis interconnected suspension, we establish the elastic force mathematical model of four-axis interconnected suspension system for heavy multi-axle vehicles. The stiffness characteristics of interconnected suspension and independent suspension system are compared and parameterized, which provides theoretical basis for reasonable design and parameter determination of stiffness for interconnected suspension system. 


\section{Elastic Force Model of Interconnected Hydro-Pneumatic Suspension}

In order to further improve driving performance of heavy multi-axle vehicles, the cylinders and accumulators of hydro-pneumatic suspension system are connected based on certain rules to achieve load balancing and anti-rolling functions. Without affecting output force characteristics of suspension system, the system is simplified and the connection diagram of four-axis interconnected hydro-pneumatic suspension system is shown in Fig.1.

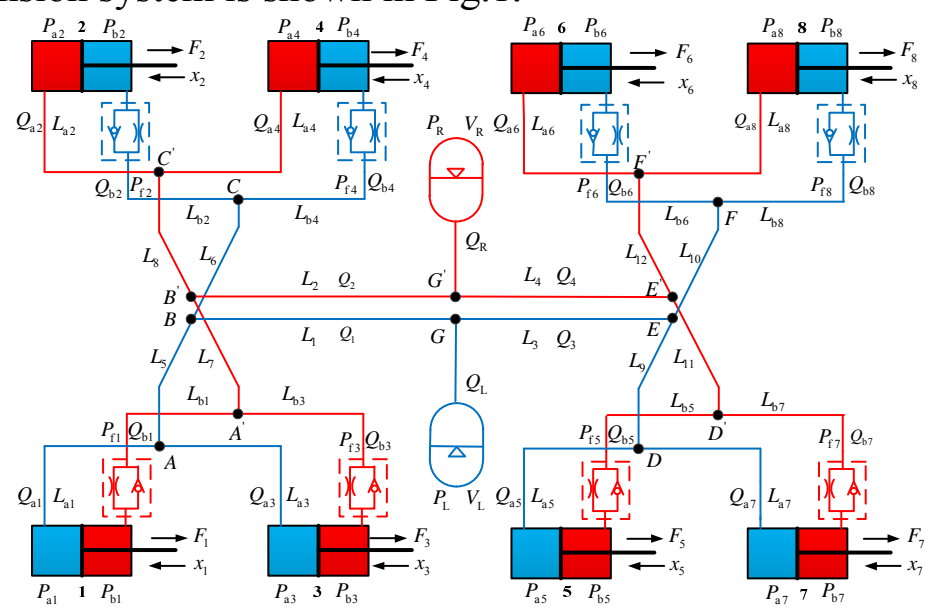

Fig. 1. Connection diagram of four-axis interconnected hydro-pneumatic suspension

Compared with independent suspension, each of four oil cylinders sharing one accumulator on the left and right sides, each cylinder is not cross-connected. In the four-axis interconnected suspension system, the rod cavities and rodless cavities are connected through hydraulic circuit, and the right and left coaxial oil cylinders are cross-connected to achieve anti-rolling function. The rod cavities are connected to each other, and the rodless cavities are connected to each other on the same side to realize balancing axial loads function. The whole suspension system is composed of two identical hydraulic system oil circuits, and an accumulator is connected in the middle of each oil circuit. When wheels are excited by vertical vibration, the movements of piston rods cause flow of oil in hydraulic circuit, so that the volume of accumulators are changed, resulting in the output elastic force of suspension system [2][8].

In order to reduce complexity of elastic force model, the following assumptions are made [6][9].

(1) The effect of temperature on oil characteristics is neglected and oil compressibility is not considered;

(2) There is no leakage of oil and gas in the whole suspension system;

(3) The cylinder is a rigid body without compression, and the geometric sizes of cylinder are the same;

(4) The effect of friction is not considered.

As shown in Fig.1, assuming that the initial volume and pressure of two accumulators under static equilibrium state are $V_{0}, P_{0}$, the volume and pressure of the left accumulator at any time $\operatorname{are} V_{\mathrm{L}}, P_{\mathrm{L}}$. The volume and pressure of the right accumulator are $V_{\mathrm{R}}, P_{\mathrm{R}}$. According to the law of gas thermodynamics[10], the equation of state of two accumulators is obtained.

$$
P_{0} V_{0}^{\gamma}=P_{\mathrm{L}} V_{\mathrm{L}}^{\gamma}=P_{\mathrm{R}} V_{\mathrm{R}}^{\gamma}
$$

Where, $\gamma$ is the adiabatic index of the gas, the whole process is an adiabatic process.

With the left accumulator as research object, the volume of accumulator at any time is related to the displacement of piston rod and initial volume of accumulator.

$$
\begin{gathered}
V_{\mathrm{L}}=V_{0}-\Delta V_{\mathrm{L}} \\
\Delta V_{\mathrm{L}}=A_{1}\left(x_{1}+x_{3}+x_{5}+x_{7}\right)-A_{2}\left(x_{2}+x_{4}+x_{6}+x_{8}\right)
\end{gathered}
$$

Similarly, the volume change of the right accumulator can be obtained.

$$
\Delta V_{\mathrm{R}}=A_{1}\left(x_{2}+x_{4}+x_{6}+x_{8}\right)-A_{2}\left(x_{1}+x_{3}+x_{5}+x_{7}\right)
$$

Based on formula $(1) \sim(4)$, we can obtain, 


$$
\begin{gathered}
P_{\mathrm{L}}=\frac{P_{0} V_{0}^{\gamma}}{\left[V_{0}+A_{2}\left(x_{2}+x_{4}+x_{6}+x_{8}\right)-A_{1}\left(x_{1}+x_{3}+x_{5}+x_{7}\right)\right]^{\gamma}} \\
P_{\mathrm{R}}=\frac{P_{0} V_{0}^{\gamma}}{\left[V_{0}+A_{2}\left(x_{1}+x_{3}+x_{5}+x_{7}\right)-A_{1}\left(x_{2}+x_{4}+x_{6}+x_{8}\right)\right]^{\gamma}}
\end{gathered}
$$

Where, the relative displacement of the vertical vibration of each cylinder piston is $x_{i}(i=1,2,3,4,5,6,7,8) . A_{1}$ is the area of the rodless cavity of cylinder, $A_{2}$ is the area of rod cavity, and the diameter of piston rod is $d$, then, $A_{1}-A_{2}=\pi d^{2} / 4$.

According to analysis of hydro-pneumatic suspension, the output force of each cylinder can be obtained as follows[5],

$$
F_{i}=P_{\mathrm{ai}} A_{1}-P_{\mathrm{bi}} A_{2}
$$

Where, $P_{\mathrm{ai}}$ is rodless cavity pressure, $P_{\mathrm{b} i}$ is rod cavity pressure.

There are pressure losses in oil flowing, which mainly include resistance loss along the way, partial pressure loss, and pressure loss caused by one-way valves and orifices. According to the flow direction of oil shown in Fig. 1, the relationship between the pressure in accumulator and the pressure in rod chamber and rodless chamber of any cylinder can be obtained.

$$
\begin{gathered}
P_{\mathrm{a} i}= \begin{cases}P_{\mathrm{L}}+\Delta P_{\mathrm{L}}+\Delta P_{\mathrm{ai}} & i=1,3,5,7 \\
P_{\mathrm{R}}+\Delta P_{\mathrm{R}}+\Delta P_{\mathrm{ai}} & i=2,4,6,8\end{cases} \\
P_{\mathrm{b} i}= \begin{cases}P_{\mathrm{R}}+\Delta P_{\mathrm{R}}+\Delta P_{\mathrm{bi}}+\Delta P_{\mathrm{fi}} & i=1,3,5,7 \\
P_{\mathrm{L}}+\Delta P_{\mathrm{L}}+\Delta P_{\mathrm{b} i}+\Delta P_{\mathrm{fi}} & i=2,4,6,8\end{cases}
\end{gathered}
$$

Where, $\Delta P_{\mathrm{L}}$ and $\Delta P_{\mathrm{R}}$ are the local pressure losses at outlet connection of the left and right accumulators; $\Delta P_{\mathrm{ai}}$ is the pressure loss along oil path to rodless cavity; $\Delta P_{\mathrm{fi}}$ is the pressure loss along oil path to rod cavity; $\Delta P_{\text {fi }}$ is the pressure loss caused by damping hole and check valve of each cylinder.

Based on formula (7) (9), the output force of cylinder can be obtained.

$$
F_{i}= \begin{cases}\left(P_{\mathrm{L}} A_{1}-P_{\mathrm{R}} A_{2}\right)+\left[\left(\Delta P_{\mathrm{L}}+\Delta P_{\mathrm{a}}\right) A_{1}-\left(\Delta P_{\mathrm{R}}+\Delta P_{\mathrm{bi}}+\Delta P_{\mathrm{fi}}\right) A_{2}\right] & i=1,3,5,7 \\ \left(P_{\mathrm{R}} A_{1}-P_{\mathrm{L}} A_{2}\right)+\left[\left(\Delta P_{\mathrm{R}}+\Delta P_{\mathrm{ai}}\right) A_{1}-\left(\Delta P_{\mathrm{L}}+\Delta P_{\mathrm{bi}}+\Delta P_{\mathrm{fi}}\right) A_{2}\right] & i=2,4,6,8\end{cases}
$$

From formula (10), it can be seen that the pressure of accumulator determines the elastic force of hydro-pneumatic suspension system. The pressure loss determines the damping force, and the output elastic force $F_{k i}$ of cylinders can be obtained as,

$$
F_{k i}= \begin{cases}P_{\mathrm{L}} A_{1}-P_{\mathrm{R}} A_{2} & i=1,3,5,7 \\ P_{\mathrm{R}} A_{1}-P_{\mathrm{L}} A_{2} & i=2,4,6,8\end{cases}
$$

According to formula (5), (6), (11), the elastic force of cylinders can be obtained.

With cylinder 1 as the research object, the output elastic force of cylinder suspension is obtained under the motion of cylinder 1 piston rod.

$$
F_{\mathrm{k} 1}=\frac{A_{1} P_{0} V_{0}^{\gamma}}{\left(V_{0}-A_{1} x\right)^{\gamma}}-\frac{A_{2} P_{0} V_{0}^{\gamma}}{\left(V_{0}+A_{2} X\right)^{\gamma}}
$$

Assuming that the pressure and volume of accumulator under static equilibrium state respectively are $P_{0}=7.2 \mathrm{MPa}, V_{0}=3.0 \mathrm{~L}$, the diameter of cylinder and piston rod respectively are $D=0.14 \mathrm{~m}, d=0.10 \mathrm{~m}$. The elastic force characteristic is shown in Fig.2, and the elastic force increases nonlinearly with the piston compression displacement. 


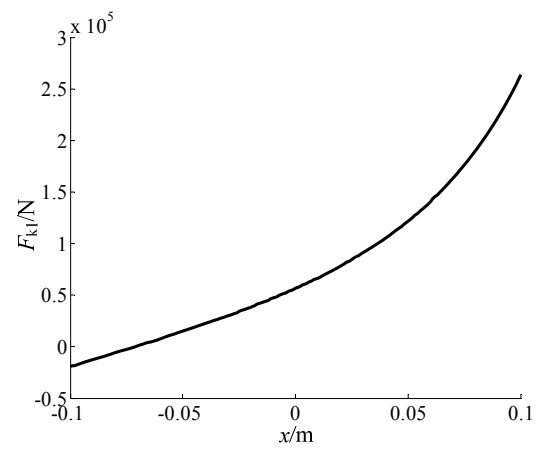

Fig. 2. The elastic force characteristic of hydro-pneumatic suspension

\section{Simulation and Test}

According to analysis of output force in the elastic force model of interconnected suspension, the modules of accumulator, cylinder, oil chamber, one-way valve and damping hole are selected in AMESim software to model the independent hydro-pneumatic suspension. The theoretical analysis of elastic force model and simulation model are verified by bench test, which lays a foundation for analysis of stiffness characteristics of interconnected suspension.Fig.3and Fig.4 are independent suspension simulation model and bench test respectively.

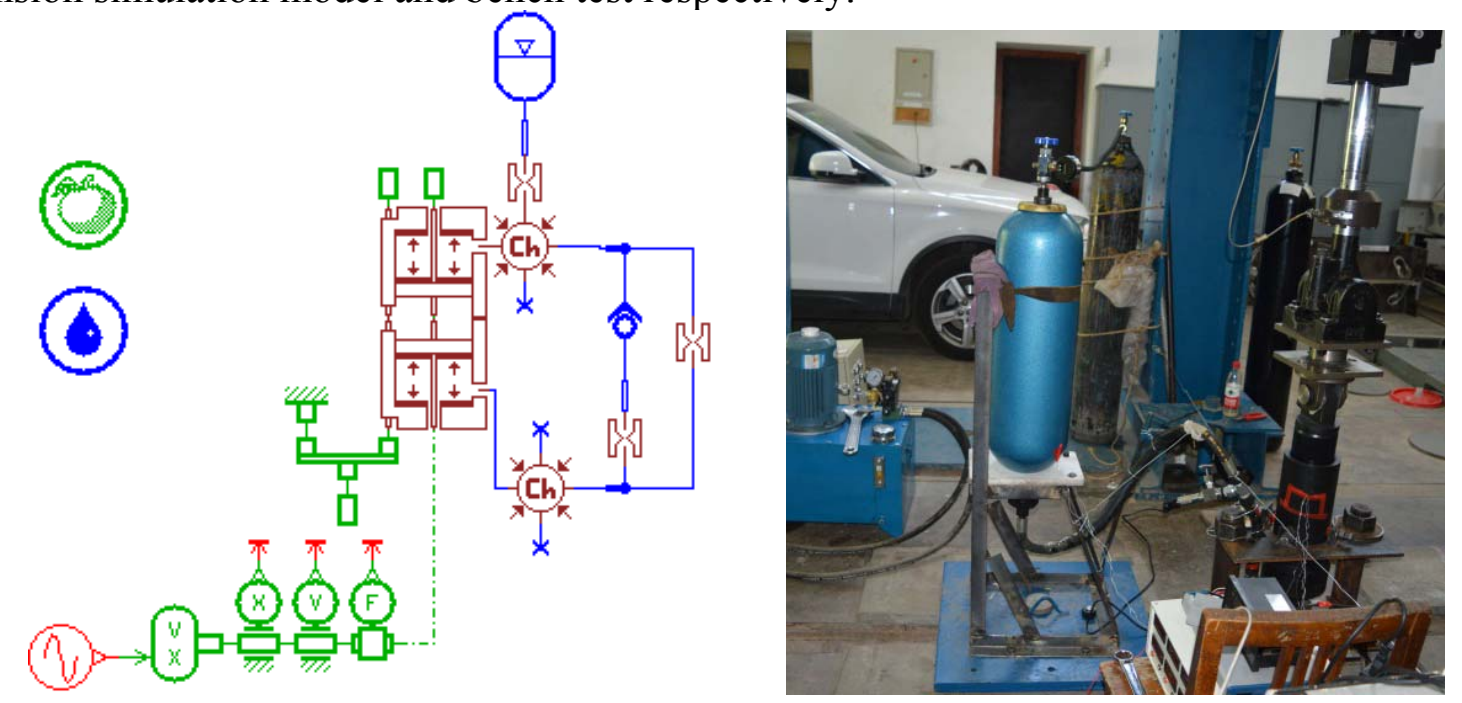

Fig.3.Independent suspension AMEsim model Fig.4.Hydro-pneumatic suspension bench test

Fig.5.shows the independent hydro-pneumatic suspension force characteristics comparison by simulation and test under sinusoidal excitation $z=0.02 \sin (2 \pi t)$. It can be seen from Fig. 5 that the difference between test and simulation curve is mainly at the two end points but the change tend is same during the process (especially in the equilibrium position). In overall stretching process of $-2 \mathrm{~mm} \rightarrow+20 \mathrm{~mm}$, the force values are between $0.24 \sim 1$. 56KN.In overall compression process of $+20 \mathrm{~mm} \rightarrow 0 \rightarrow-20 \mathrm{~mm}$, the force values are between $0 \sim 4.05 \mathrm{KN}$. The simulation values in the overall stretching process are slightly smaller than the test values, but the simulation values of the overall compression process are slightly larger than experimental values, the reason is that the friction force, the weight of cylinder and fixture are ignored in the force characteristics model. The overall data deviation is within the margin of error, which can be used as the basis for theoretical analysis of the elastic force model of interconnected hydro-pneumatic suspension. 


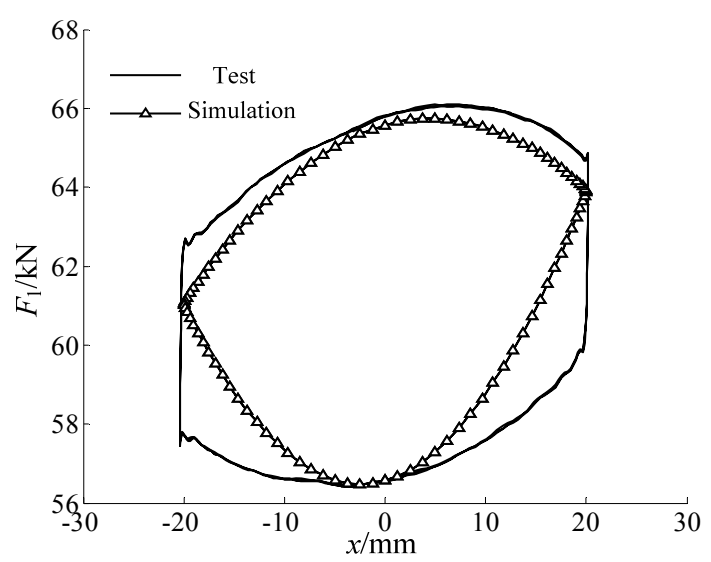

Fig. 5. The output force characteristics comparison

\section{Analysis of Stiffness Characteristics of Interconnected Suspension System}

\subsection{Stiffness Characteristics Under Vertical Condition}

Only one cylinder is subjected to vertical excitation under single wheel vertical excitation condition. Taking cylinder 1 as the research object, assuming that $x_{1}=x, x_{i}=0, i=2, \ldots, 8$ according to the definition of stiffness, the stiffness of hydro-pneumatic suspension can be obtained under single wheel vertical excitation condition by taking derivation of formula (12) with respect to the displacement $x[8]$.

$$
K_{1}=\frac{\partial F_{\mathrm{k} 1}}{\partial x}=\gamma P_{0} V_{0}^{\gamma}\left(\frac{A_{1}^{2}}{\left(V_{0}-A_{1} x\right)^{\gamma+1}}+\frac{A_{2}^{2}}{\left(V_{0}+A_{2} x\right)^{\gamma+1}}\right)
$$

As for the independent hydro-pneumatic suspension system, each cylinder is independent, and four cylinders on the left and right sides share an accumulator. Under single wheel vertical excitation condition, the output elastic force and vertical stiffness are,

$$
\begin{gathered}
F_{\mathrm{k} 1}^{*}=\frac{A_{0} P_{0} V_{0}^{\gamma}}{\left(V_{0}-A_{0} x\right)^{\gamma}} \\
K_{1}^{*}=\frac{\partial F_{\mathrm{k} 1}{ }^{*}}{\partial x}=\frac{A_{0}{ }^{2} \gamma P_{0} V_{0}^{\gamma}}{\left(V_{0}-A_{0} x\right)^{\gamma+1}}
\end{gathered}
$$

The stiffness characteristics under single wheel vertical excitation condition are compared as shown in Fig.6.

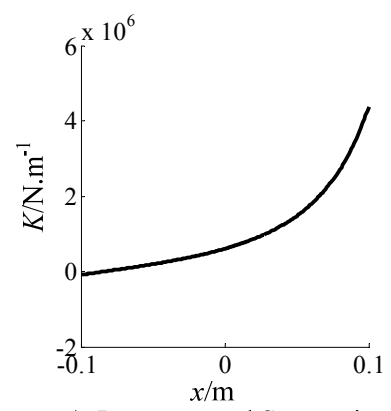

a) Interconected Suspension

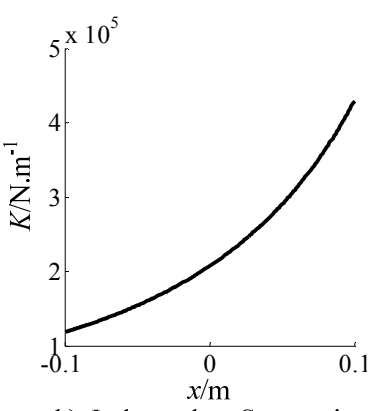

b) Independent Suspension

Fig. 6. Stiffness characteristics comparison of suspension system

From the stiffness characteristics comparison in Fig.6, it can be seen that the stiffness increases during the compression process, which impedes the relative movement of piston rod. The stiffness of interconnected suspension system is much larger than that of independent suspension system. Its nonlinearity is also superior to that of independent suspension.

Assuming that the relative displacement of all pistons is the same under vertical excitation condition, that is, $x_{i}=x(i=1,2 \ldots, 8)$, according to the elastic force model, the elastic force of the suspension is obtained. 


$$
F_{\mathrm{k} i}=\frac{A_{0} P_{0} V_{0}^{\gamma}}{\left(V_{0}-4 x A_{0}\right)^{\gamma}}(i=1,2 \ldots, 8)
$$

Under this condition, the elastic force characteristics of interconnected suspension and independent suspension are the same, and the vertical stiffness is

$$
K_{2}=\frac{\partial F_{\mathrm{k} i}}{\partial x}=\frac{4 \gamma A_{0}^{2} P_{0} V_{0}^{\gamma}}{\left(V_{0}-4 A_{0} x\right)^{\gamma+1}}
$$

Formula (17) reflects the vertical stiffness characteristics of interconnected suspension under vertical excitation condition. The main influence parameters are the initial volume, initial pressure of accumulator and piston rod diameter. Fig. 7 9 show the effect of each parameter on vertical stiffness.

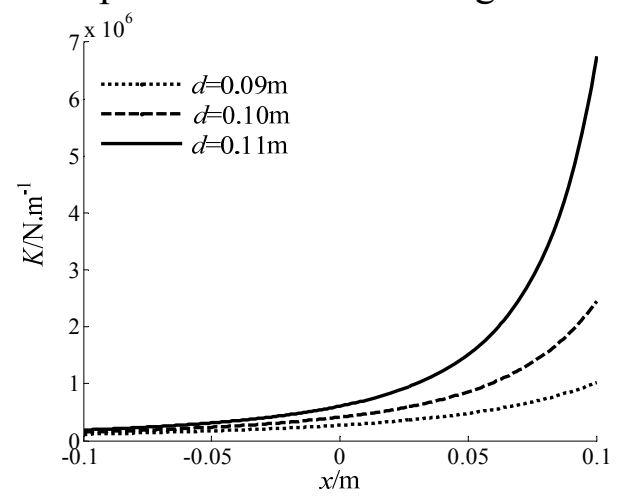

Fig. 7. Vertical stiffness characteristics under different diameter of piston rod

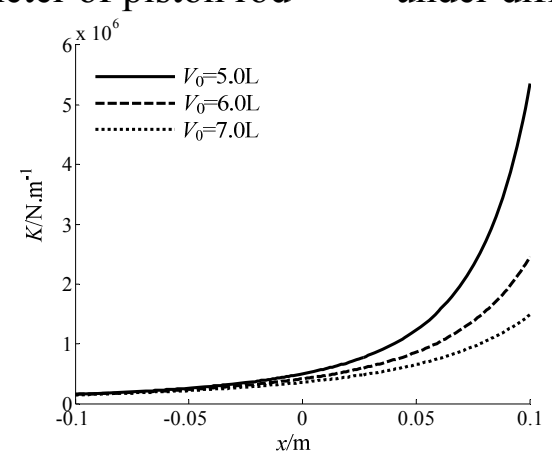

Fig. 9. Vertical stiffness characteristics under different initial volume

As can be seen from Fig.7 9, when suspension displacement is small, the stiffness is small, which is beneficial to its smoothness. As compression displacement increases, the stiffness of interconnected suspension system increases nonlinearly. As the diameter of piston rod increases, the vertical stiffness increases, the nonlinear characteristics increase. The initial pressure of accumulator has less influence on vertical stiffness under this working condition, and the initial pressure increases, and the vertical stiffness increases, and the nonlinear characteristics do not change significantly. As the initial volume of accumulator increases, the vertical stiffness decreases.

\subsection{Stiffness Characteristics Under Rolling Condition}

The piston displacement of the right and left suspension cylinder is opposite under rolling condition. Assuming that the roll angle is $\varphi$ and the installation distance of two cylinders between left and right is $B$, the cylinder displacement $x_{1,3,5,7}=\frac{B}{2} \varphi, x_{2,4,6,8}=-\frac{B}{2} \varphi$, and the roll moment of suspension is obtained as follows,

$$
M_{\varphi}=\frac{B}{2}\left(F_{\mathrm{k} 1}+F_{\mathrm{k} 3}+F_{\mathrm{k} 5}+F_{\mathrm{k} 7}-F_{\mathrm{k} 2}-F_{\mathrm{k} 4}-F_{\mathrm{k} 6}-F_{\mathrm{k} 8}\right)
$$

Based on formula 5, 6, 1 land 18, the roll moment of interconnected suspension system can be obtained. 


$$
M_{\varphi}=\frac{2 B\left(A_{1}+A_{2}\right) P_{0} V_{0}^{\gamma}}{\left[V_{0}-2 B \varphi\left(A_{1}+A_{2}\right)\right]^{\gamma}}-\frac{2 B\left(A_{1}+A_{2}\right) P_{0} V_{0}^{\gamma}}{\left[V_{0}+2 B \varphi\left(A_{1}+A_{2}\right)\right]^{\gamma}}
$$

However, the roll moment of independent suspension is

$$
M_{\varphi}^{*}=\frac{2 B A_{0} P_{0} V_{0}^{\gamma}}{\left(V_{0}-2 A_{0} B \varphi\right)^{\gamma}}-\frac{2 B A_{0} P_{0} V_{0}^{\gamma}}{\left(V_{0}+2 A_{0} B \varphi\right)^{\gamma}}
$$

With taking the derivation of roll moment with respect to roll angle $\varphi$, the roll stiffness of interconnected suspension and independent suspension can be obtained.

$$
\begin{gathered}
K_{\varphi}=\frac{\partial M_{\varphi}}{\partial \varphi}=\frac{4 B^{2}\left(A_{1}+A_{2}\right)^{2} \gamma P_{0} V_{0}^{\gamma}}{\left[V_{0}-2 B \varphi\left(A_{1}+A_{2}\right)\right]^{\gamma+1}}+\frac{4 B^{2}\left(A_{1}+A_{2}\right)^{2} \gamma P_{0} V_{0}^{\gamma}}{\left[V_{0}+2 B \varphi\left(A_{1}+A_{2}\right)\right]^{\gamma+1}} \\
K_{\varphi}^{*}=\frac{\partial M_{\varphi}^{*}}{\partial \varphi}=\frac{4 B^{2} A_{0}^{2} \gamma P_{0} V_{0}^{\gamma}}{\left(V_{0}-2 B \varphi A_{0}\right)^{\gamma+1}}+\frac{4 B^{2} A_{0}^{2} \gamma P_{0} V_{0}^{\gamma}}{\left(V_{0}+2 B \varphi A_{0}\right)^{\gamma+1}}
\end{gathered}
$$

Fig.10 and 11 are the roll moment and roll stiffness characteristics comparison of interconnected suspension and independent suspension system. It can be seen that the roll moment and roll stiffness of interconnected suspension system are much larger than that of independent suspension, and the nonlinearity of roll stiffness is also better than that of independent suspension. For interconnected suspension system, when the roll angle $|\varphi| \leq 3^{\circ}$, the roll stiffness is small and can keep a certain value, that is, the roll moment increases linearly at the equilibrium position. When the roll angle $|\varphi|>3^{\circ}$, the roll stiffness increases rapidly with the increase of roll angle. When the roll angle of vehicle is large, it can provide a very large roll stiffness and improve stability of vehicles. For independent suspension system, the roll stiffness increases as parabola, and its nonlinear characteristic is poor.

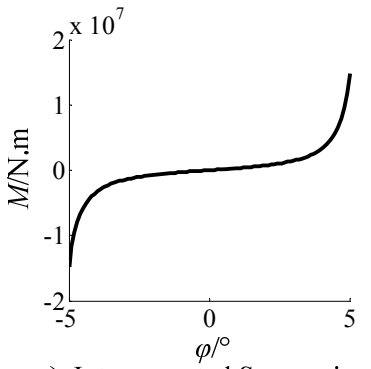

a) Interconected Suspension

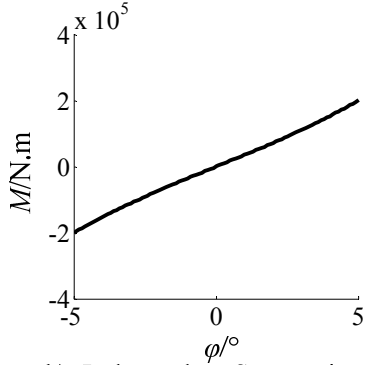

b) Independent Suspension

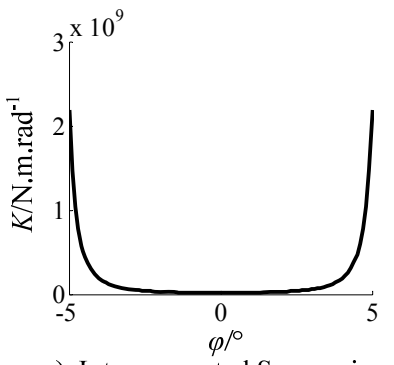

a) Interconnected Suspension

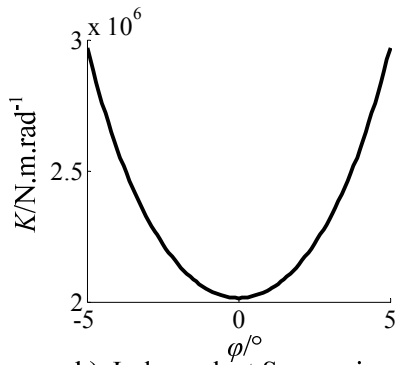

b) Independent Suspension

Fig. 10. Roll moment characteristics comparison Fig. 11. roll stiffness characteristics comparison

Formula (21) is an expression for the roll stiffness of interconnected suspension system. The roll stiffness of interconnected suspension system is mainly affected by the diameter of cylinder, the diameter of piston rod, the initial pressure and initial volume of accumulator, the installation distance of cylinders between left and right. Fig.12 16 show the influence of different parameters on roll stiffness.

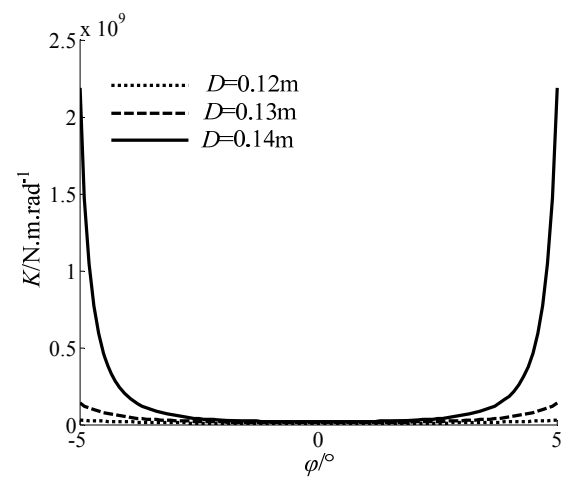

Fig.12.Roll stiffness characteristics under different cylinder diameter

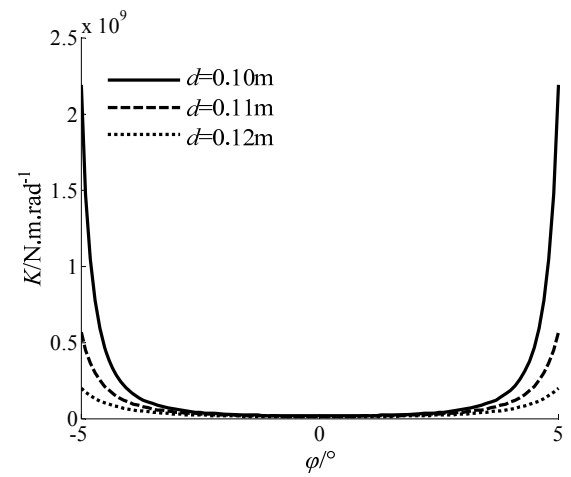

Fig. 13. Roll stiffness characteristics under different piston rod diameters 


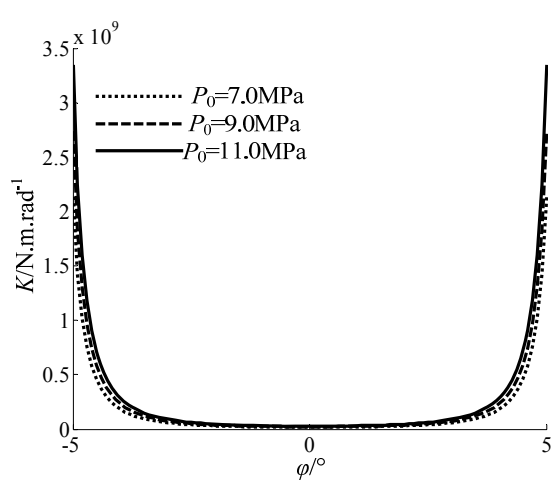

Fig.14.Roll stiffness characteristics under different initial pressure

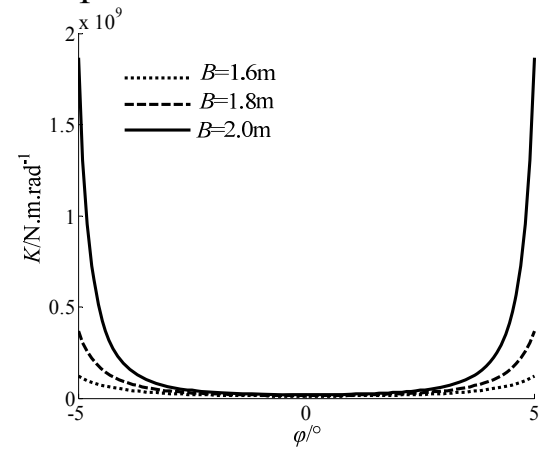

Fig. 16. Roll stiffness characteristics under different installation distance

From Fig. $12 \sim 16$, it can be seen that roll stiffness of interconnected suspension system increases and the nonlinear characteristic is enhanced with the increase of the diameter of cylinder. The smaller the diameter of piston rod is, the larger the roll stiffness is, and the nonlinear characteristic is obvious. With the increase of initial pressure of accumulator, the roll stiffness increases, but the influence on nonlinear characteristics is not obvious. The smaller the initial volume of accumulator, the greater the roll stiffness and the more obvious the nonlinear characteristics. Increasing the installation distance of cylinders between left and right will increase roll stiffness and enhance nonlinearity.

\section{Conclusion}

The elastic force model of four-axle interconnected suspension system for heavy multi-axle vehicle is established, and the simulation and test are carried out. The stiffness characteristics of interconnected suspension and independent suspension are compared and analyzed under different working conditions. The following conclusions are drawn:

(1) Compared with independent suspension, the interconnected suspension system can provide a larger and more nonlinear vertical stiffness under single wheel vertical excitation condition. And under rolling condition, it can provide a larger roll stiffness and improve stability of vehicles. When the roll angle is small, the roll stiffness is almost constant and relatively small, ensuring the riding comfort of vehicles.

(2) Different parameters have different effects on vertical and roll stiffness characteristics. The vertical stiffness increases with the increase of the diameter of the piston rod, the increase of the initial pressure and the decrease of the initial volume of accumulator under static equilibrium state. The roll stiffness increases with the increase of the diameter of the cylinder, the decrease of the diameter of the piston rod, the decrease of initial volume and the increase of the initial pressure of accumulator, and the increase of the installation distance of two cylinders between left and right.

\section{References}

[1]. PENG Mo, ZHOU Liangsheng, YUE Jingtao, et al.Multi-Axle Motor Vehicles[M].Beijing:China Machine Press,2014. 
[2]. ZHANG Junwei,YANG Bo,LI Hongbiao,et al.Research on Modeling and Characteristics of Interconnected Hydropneumatic Suspension in Multiple Modes[J].Chinese Journal of Automotive Engineering,2016,6(3):212-222.

[3]. CAO Dongpu, RAKHEJA S, SU Chunyi. Roll-and Pitch-plane Coupled Hydro-pneumatic Suspension:Part I:Feasibility Analysis and Suspension Properties[J]. Vehicle System Dynamics, 2010, 48(3):361-386.

[4]. W Smith,N Zhang. Recent Developments in Passive Interconnected Vehicle Suspension [J].Front.Mech.Eng. China, 2010, 5(1): 1-18.

[5]. WANG Zengquan,SHEN Yanhua, YANG Jue.Mathematical Model and Characteristics Analysis of Interconnected Hydro-pneumatic suspension[J].Transactions of the Chinese Society of Agricultural Engineering, 2012,28(5):60-65.

[6]. CHEN Shengzhao,ZHONG Yixu,ZHANG Bangji,et al.Influence of Key Parameters of Hydraulically Interconnected Suspension on Vehicle Dynamics and Experimental Validation[J].Jourual of Mechanical Engineering, 2017,53(14):39-48.

[7]. DING Fei,ZHANG Nong, HAN Xu. Modeling and Modal analysis of Multi-Body Truck System Fitted with Hydraulically Interconnected Suspension [J]. Journal of Mechanical Engineering, 2012, 48(6):116-123.

[8]. SUN Yanbin.Research on Characteristics of Hybrid-interconnected Hydro-pneumatic Suspension and Analysis of Ride Comfort [D].Dalian:Dalian University of Technology,2016.

[9]. ZHANG Junwei,CHEN Sizhong,WU Zhicheng,et al. Coupling Characteristics of Stiffness and Damping of Multi-branch Interconnected Hydro-pneumatic Suspension[J].Journal of Sichuan Military Engineering,35(5):80-85.

[10]. LIU Xiaohong, XU Tao.Fluid Mechanincs and Thermal Engineering[M].Beijing:China Machine Press,2012. 